\title{
Observations of Eclipses of the Satellites of Jupiter, made at Washington during the Opposition of 1879.
}

By D. P. Todd, Assistant Nautical Almanac.

From my series of observations of the phenomena of the Satellites of Jupiter during the opposition of 1879, I select the eclipses for present publication. The instruments used in making these observations are the property of the Nautical Almanac office.

(1) An equatorial refractor of $3^{\text {incli } 5}$ aperture, and a focal length of forty-eigth inches.

(2) An equatorial refractor of $3^{\text {inch }} 0$ aperture, and a focal lenght of forty-four inches.

The scale of weights embraces 1 to 5, as follows: - 1 uncertain, 2 indifferent, 3 average, 4 good, 5 excellent.

Not having been able to use the method of observing with the occulting-bar in the observations made before August 30th, it was not employed after that time, in order that the conditions of observing might be kept as nearly as possible symmetrical with reference to the date of opposition.

I am indebted to Professor J. R. Eastman, U. S. Navy, for some of the data necessary in reducing my chronometer-corrections to the standard time of the Naval Observatory.

\begin{tabular}{|c|c|c|c|c|c|c|c|c|}
\hline \multicolumn{2}{|c|}{ Date } & 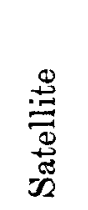 & 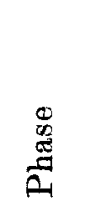 & $\begin{array}{l}\text { Washington } \\
\text { Mean Time }\end{array}$ & 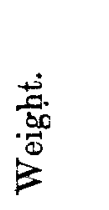 & 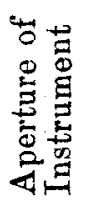 & 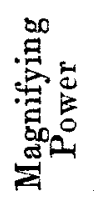 & $\mathrm{R}$ e $\mathrm{m}$ a $\mathrm{rk}$ \\
\hline May & 30 & II & Dis. & $\left\{15^{\mathrm{h}} 10^{\mathrm{m}} 43^{\mathrm{s}}\right.$ & - & 3.0 & 120 & First diminution of brightness. \\
\hline \multirow[t]{12}{*}{ August } & 2 & IV & - & $\begin{array}{lrr}9 & 1 & 39.7\end{array}$ & 2 & 3.0 & 65 & Moon $0^{d} 8$ past full, and $21^{\circ}$ from Jupiter. \\
\hline & & IV & $\mathrm{Re}_{\mathrm{e}}$ & $(132259.1$ & 4 & 3.0 & 65 & Moon $1^{\mathrm{d}} 0$ past full, and $19^{\circ}$ from Jupiter. \\
\hline & & IV & Re. & 13368 & - & 3.0 & 65 & Full brightness. \\
\hline & 9 & II & Dis. & $\left\{\begin{array}{lll}14 & 17 \quad 8\end{array}\right.$ & 2 & 3.0 & 65 & First diminution of brightness. \\
\hline & & & Dis. & $14 \quad 25 \quad 11.8$ & 3 & 3.0 & 65 & Moon $1 \mathrm{~d} 0$ past full, and $18^{\circ}$ from Jupiter. \\
\hline & 12 & I & - & $\left\{\begin{array}{l}95213 \\
0\end{array}\right.$ & 2 & 3.0 & 65 & First diminution of brightness. \\
\hline & & & 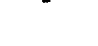 & 19.5519 .8 & 2.5 & 3.0 & 65 & Last seen. \\
\hline & 19 & I & - & 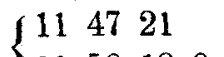 & - & 3.0 & 120 & First diminution of brightness. \\
\hline & 18 & 1 & - & 115018.0 & 2.5 & 3.0 & 120 & Last seen. \\
\hline & 20 & III & - & 83532.4 & 2 & 3.0 & 65 & $\begin{array}{l}\text { Satellites II and III were so near together } \\
\text { that the observation was difficult. }\end{array}$ \\
\hline & 20 & II & - & 85158.0 & 3 & 3.0 & 65 & $\begin{array}{l}\text { A suspicion that the satellite was seen } \\
12^{\text {s later. }}\end{array}$ \\
\hline & 28 & I & - & 81354.7 & 2.5 & 3.0 & 120 & $\begin{array}{l}\text { Jupiter only two days from opposition, and } \\
\text { the satellite disappeared very near the } \\
\text { limb of the planet. Moon } 27 \text { before } \\
\text { full, and } 36^{\circ} \text { from Jupiter. }\end{array}$ \\
\hline \multirow[t]{5}{*}{ Sept. } & 4 & I & Re. & 12.2329 .5 & 3 & 3.5 & 75 & $\begin{array}{l}\text { Moon } 3^{\mathrm{d}} 6 \text { before last quarter, and } 56^{\circ} \text { from } \\
\text { Jupiter. }\end{array}$ \\
\hline & 6 & I & - & 65325.4 & 1 & 3.0 & 65 & $\begin{array}{l}\text { Twilight still visible. Sky somewhat smoky, } \\
\text { and seeing bad. }\end{array}$ \\
\hline & 25 & III & - & $\left(\begin{array}{lll}8 & 4 & 0.7\end{array}\right.$ & 4 & 3.5 & 75 & $\begin{array}{l}\text { Moon } 3^{\mathrm{d} 2} \text { after first quarter, and } 23^{\circ} \text { from } \\
\text { Jupiter. }\end{array}$ \\
\hline & & & & $8 \quad 81055$ & - & 3.5 & 75 & Full brightness. \\
\hline & 27 & $\mathbf{I}$ & - & $\left\{\begin{array}{lll}12 & 38 & 1.8 \\
12 & 40 & 52\end{array}\right.$ & -3 & $\begin{array}{l}3.5 \\
3.5\end{array}$ & $\begin{array}{l}140 \\
140\end{array}$ & $\begin{array}{l}\text { Moon } 2^{\mathrm{d} 2} \text { before full, and } 7^{\circ} \pm \text { from Jupiter. } \\
\text { Full brightness. }\end{array}$ \\
\hline
\end{tabular}


1879

$$
\text { 递 }
$$

Washington Mean Time

Sept. 29 $7^{\mathrm{h}} 8 \mathrm{~m} 2 \mathrm{~s} 0$

Re.

$\begin{cases}742^{\mathrm{s}} 0 & ? \\ 71054 & 2\end{cases}$

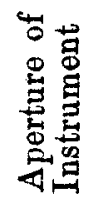

3.5

3.5

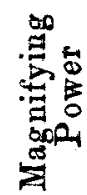

140 140

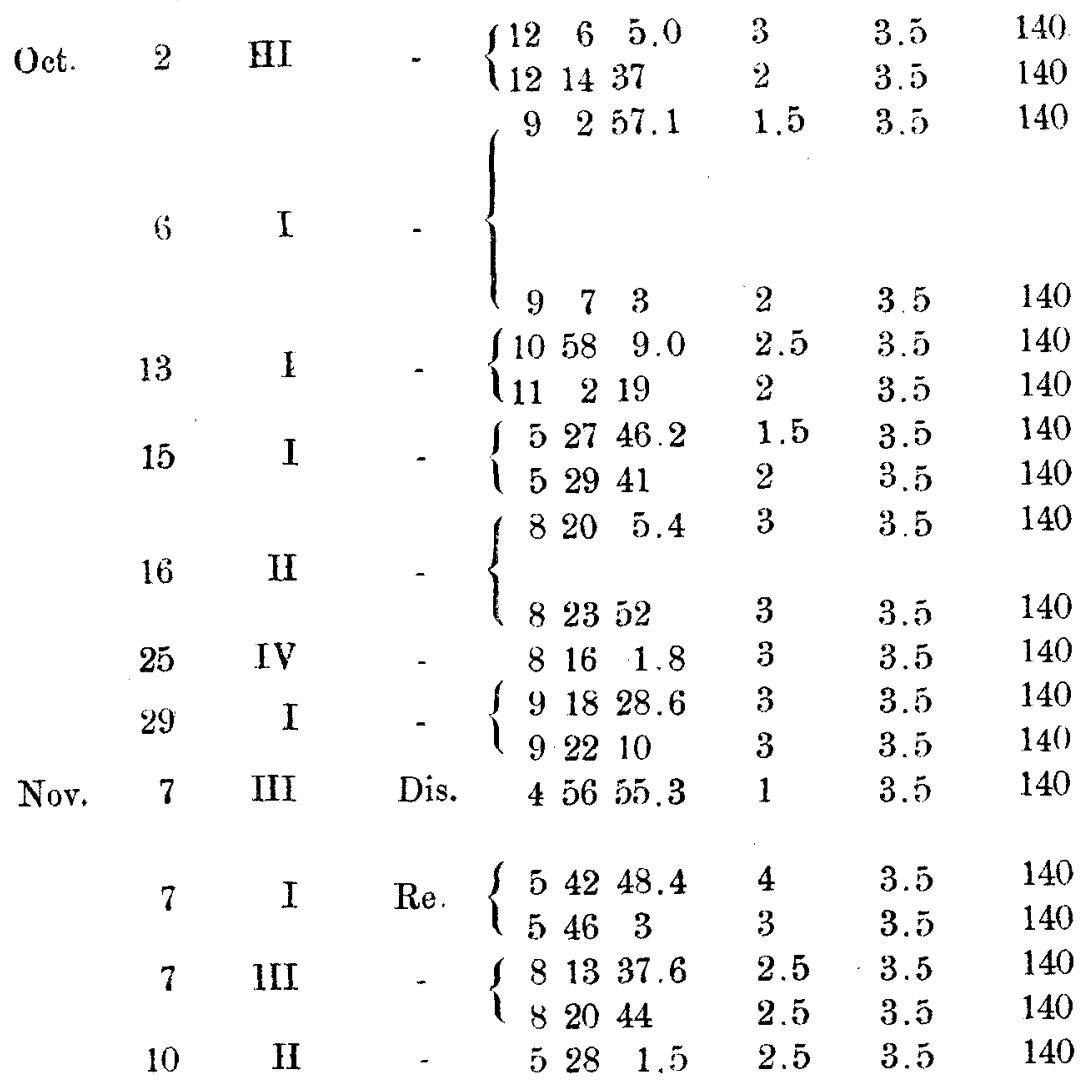

$3.5 \quad 140$

3.5

3.5

3.5
140 Full brightness.

140 First diminution of brightness.

140 Disappearance. Portions of the sky were dimmed by a thin haziness. I do not think, however, that it rested over the planet at the moment of observation.

First seon.

Full brightness. At the time recorded "first seen ", the planet was behind passing clouds which were almost dense enough to obscure the three other satellites. The first satellite was already too far past actual reappearance for this time to be assigned by estimation even: it was fully onethird its full brightness.

Moon $2^{\mathrm{d}} 8$ past full, and $67^{\circ}$ from Jupiter. Full brightness.

First seen. Came into view quite suddenly. I think the planet was behind thin flying clouds which passed off at the time recorded. Seeing not good.

Full brightness.

Full brightness.

First seen. Very bright twilight.

Full brightness.

First seen. Possibly an extremely thin haze over the sky.

Full brightness.

Moon $4^{\mathrm{a}} \mathrm{O}$ before full, and $14^{\circ} \pm$ from Jupiter.

Moon at full, and $64^{\circ}$ from Jupiter.

Full brightness.

Timeof iobservation near sunset. Observation quite uncertain.

Full brightness.

Full brightness.

Not long after sunset. Possibly a thin cloud rested over the planet at the moment of observation. The reappearance was quite definite.

14 III Dis. $\left\{\begin{array}{llllll}7 & 41 & 34 & 2 & 3.5 & \\ 8 & 53 & 19 & 3 & 3.5 & 140 \\ 8 & 59 & 38.4 & 3 & 3.5 & 140\end{array}\right.$




\begin{tabular}{|c|c|c|c|c|c|c|c|}
\hline 187 & & 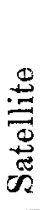 & 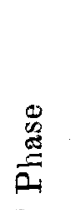 & $\begin{array}{l}\text { Washington } \\
\text { Mean Time }\end{array}$ & $\begin{array}{l}\vec{\Delta} \\
.00 \\
\overrightarrow{0}\end{array}$ & 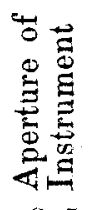 & 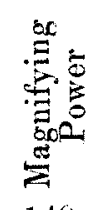 \\
\hline Nor. & 21 & $I$ & Re. & $9+34^{m} 31^{5} 3$ & 2.5 & 3.5 & 140 \\
\hline & 24 & II & - & $1043 \quad 14.9$ & 1 & 3.5 & 140 \\
\hline & & I & - & f 55849.5 & 3 & 3.5 & \\
\hline & 30 & 1 & - & $\left\{\begin{array}{lll}6 & 2 & 9\end{array}\right.$ & $\ddot{3}$ & 3.5 & \\
\hline & & & & $\left(\begin{array}{lll}7 & 55 & 28.0\end{array}\right.$ & 1 & 3.5 & \\
\hline Dec. & 7 & I & - & & & & \\
\hline & & & & $757 \quad 7$ & 1 & 3.5 & \\
\hline & 12 & $\mathrm{H}$ & - & $\begin{array}{lll}516 & 26.1\end{array}$ & 2.5 & 3.5 & \\
\hline & 30 & I & - & 81038.2 & 1 & 3.5 & \\
\hline 188 & & & & $\int \begin{array}{lll}629 & 24.9\end{array}$ & 3 & 35 & \\
\hline Jan. & 15 & $I$ & - & $\left\{\begin{array}{l} \\
63253\end{array}\right.$ & 3 & 3.5 & \\
\hline Febr. & 7 & I & - & $\begin{array}{lll}6 & 43 & 0.8\end{array}$ & 1.5 & 3.5 & \\
\hline
\end{tabular}

Washington, 13. February 1880.

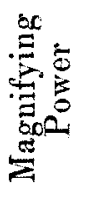

$$
\mathrm{R} \text { c mark. }
$$

Moon 1.3 past first quarter, and $13^{\prime \prime}+$ from Jupiter.

Moon $4^{4} 4$ past first quarter, and $49^{\circ}$ from Jupiter. Just as I was expecting to observe the reappearance of the satellite, the planet passed from a perfectly clear sky into a rising cloud-bank, which, though not very dense, must have retarded the eclipse not far from forty seconds.

Full brightness.

A dense haze over the sky. Jupiter appeared to shine at about one-third its usual brilliancy in a clear sky.

Full brightness.

Considerable twilight.

Clouds were passing over the planet, and it shone with about onc-half its usual brilliancy.

Moon $3^{\mathrm{d}} 3$ before first quarter, and about $7^{\circ}$ from Jupiter.

Full brightness.

Planet rather low, and very faint twilight underneath it.

$$
\text { I. P. Todd. }
$$

\section{'Todes-Anzeige.}

Am 5. Februar starb der langjährige erste Assistent der Sternwarte in Madras, Herr Chintamanay Ragoonatha Charry. Seit mehr als 35 Jabren an der dortigen Sternwarte angestellt, hat er dreimal die Leitung derselben in andere Hände übergehen sehen, und sich die Achtung eines jeden seiner Vorgesetzten durch Zuverlässigkeit im Beobachten und Rechnen, und eine selbsterworbene tüchtige Konntniss auf mathematischem und astronomischem Gebiete zu erwerben gewusst. In den Monthly Notices of the R. Astr. Soc. Vol. 19 findet sich ein Artikel des Verstorbecen über Bestimmung der persönlichen Gleichungen, ausserdem machte er sich bekannt durch Entdeckung zweier veränderlicher Sterne, $R$ Reticuli im Jahre 1867, und V Cephei im Jahre 1878, und eine grosse Anzahl seit dem Jahre 1862 von ihm ausgeführter astronomischer Beobachtungen harrt noch der Veröffentlichung. Ein in neueren Zeiten seltenes Beispiel von wissenschaftlicher Befähigung und ausdauerndem Fleisse bei einem Eingebornen Indiens, hat der Verstorbene sich durch Abfassung populärer Aufsätze in einheimischen Kalendern und öffentliche Vorträge um die Verbreitung richtiger Anschauungen unter seinen noch grossentheils in astrologischem Aberglauben befangenen Landsleuten nicht geringe Verdienste erworben.

\section{Berichtigung.}

Astr. Nachr. Nr. 2298 S. 282 Z. 28 v. unten statt "von Olbers" lies "vor Olbers". 